\title{
Levels of high-density lipoprotein cholesterol (HDL-C) among children with steady-state sickle cell disease
}

Magda O Seixas ${ }^{1,2}$, Larissa C Rocha ${ }^{1,3}$, Mauricio B Carvalho ${ }^{2}$, Joelma F Menezes ${ }^{1,2}$, Isa M Lyra ${ }^{3,4}$,
Valma ML Nascimento ${ }^{3}$, Ricardo D Couto ${ }^{2}$, Ájax M Atta ${ }^{2}$, Mitermayer G Reis ${ }^{1}$, Marilda S Goncalves ${ }^{1,2^{*}}$

\begin{abstract}
Background: The search for sickle cell disease (SCD) prognosis biomarkers is a challenge. These markers identification can help to establish further therapy, later severe clinical complications and with patients follow-up. We attempted to study a possible involvement of levels of high-density lipoprotein cholesterol (HDL-C) in steadystate children with SCD, once that this lipid marker has been correlated with anti-inflammatory, anti-oxidative, antiaggregation, anti-coagulant and pro-fibrinolytic activities, important aspects to be considered in sickle cell disease pathogenesis.
\end{abstract}

Methods: We prospectively analyzed biochemical, inflammatory and hematological biomarkers of 152 steady-state infants with SCD and 132 healthy subjects using immunochemistry, immunoassay and electronic cell counter respectively. Clinical data were collected from patient medical records.

Results: Of the 152 infants investigated had a significant positive association of high-density lipoprotein cholesterol with hemoglobin $(P<0.001)$, hematocrit $(P<0.001)$ and total cholesterol $(P<0.001)$ and a negative significant association with reticulocytes $(P=0.046)$, leukocytes $(P=0.015)$, monocytes $(P=0.004)$ and platelets $(P=0.005)$, bilirubins [total bilirubin $(P<0.001)$, direct bilirubin $(P<0.001)$ and indirect bilirubin $(P<0.001$ ], iron $(P<0.001)$, aminotransferases [aspartate aminotransferase $(P=0.004)$, alanine aminotransferase $(P=0.035)$ ], lactate dehydrogenase $(P<0.001)$, urea $(P=0.030)$, alpha 1-antitrypsin $(P<0.001)$, very low-density lipoprotein cholesterol $(P=0.003)$, triglycerides $(P=0.005)$ and hemoglobin $S(P=0.002)$. Low high-density lipoprotein cholesterol concentration was associated with the history of cardiac abnormalities $(P=0.025)$, pneumonia $(P=0.033)$ and blood transfusion use $(P=0.025)$. Lipids and inflammatory markers were associated with the presence of cholelithiasis.

Conclusions: We hypothesize that some SCD patients can have a specific dyslipidemic subphenotype characterized by low HDL-C with hypertriglyceridemia and high VLDL-C in association with other biomarkers, including those related to inflammation. This represents an important step toward a more reliable clinical prognosis. Additional studies are warranted to test this hypothesis and the probably mechanisms involved in this complex network of markers and their role in SCD pathogenesis.

\footnotetext{
* Correspondence: mari@bahia.fiocruz.br

'Laboratório de Patologia e Biologia Molecular, Centro de Pesquisa Gonçalo Moniz, Fundação de Pesquisa Oswaldo Cruz (FIOCRUZ), Salvador, Bahia, Brasil Full list of author information is available at the end of the article
} 


\section{Background}

Sickle cell disease (SCD) clinical outcomes vary widely from mild to severe and the disease has been associated with multi-organ damage and risk of early mortality $[1,2]$. Acute and chronic clinical manifestations of SCD include vaso-occlusive pain episodes (VOE), impaired blood flow as a result of intravascular sickling in capillaries and small vessels, inflammation processes and high susceptibility to infection. Researchers have found a complex network of associations among laboratory analyses and clinical events predicting a probably risk of death $[1,3,4]$.

The sickle cell disease vaso-occlusive phenomenon has been described as a complex event with the participation of stressed reticulocytes, sickled erythrocytes, leukocytes, platelets and endothelium activation [2,5-8]. Reactive oxygen species (ROS), scavenger molecules and nitric oxide $(\mathrm{NO})$ play important roles as regulators of vascular homeostasis in SCD pathogenesis [9].

Several biomarkers have been associated with SCD clinical prognosis; some, such as fetal hemoglobin ( $\mathrm{HbF})$ concentration, leukocytes count and reticulocyte count are considered to be classic [2,5]. Recently, serum lactate dehydrogenase (LDH), a well-known marker of intravascular hemolysis, was described as a biomarker of prognosis in SCD [10]. It has been associated with nitric oxide resistance, priapism, leg ulceration, pulmonary hypertension, and death in SCD patients [11].

We conducted a prospective study to investigate highdensity lipoprotein cholesterol (HDL-C) levels, including also determination of total cholesterol, low-density lipoprotein cholesterol (LDL-C), very low-density lipoprotein cholesterol (VLDL-C) and triglycerides to test the hypothesis that they can be used as a marker of prognosis among steady-state sickle cell disease children. This potential biomarker and their association with others laboratory determination and medical history were investigated in order to identify sub-phenotypes associated with the disease.

\section{Subjects and Methods}

\section{Subjects and Controls}

Of 152 steady-state SCD children from Salvador city, state of Bahia, in Brazil were prospectively analyzed for laboratory (biochemical and hematological) markers. Brazil is the largest country in South America, with one of the most heterogeneous populations due to several waves of immigration that have resulted in cultural, socioeconomic, and ethnic diversity in different geographic regions. Salvador is the largest city in Bahia, a Northeastern Brazilian state. Among the local population, $86 \%$ is of African origin, and Salvador has the highest incidence of SCD in Brazil [12].
The study was conducted from March 2007 to November 2008 and included patients from the Fundação de Hematologia e Hemoterapia do estado da Bahia (HEMOBA), a reference center attending to sickle cell disease patients who are seen for routine visits at the outpatient clinic. The study also included 132 healthy children randomly selected from the Clinical Laboratory of the Faculdade de Farmácia da Universidade Federal da Bahia (UFBA); these were matched to cases by age, gender and African ethnic origin as a control group. The study was approved by the Fundação de Pesquisa Oswaldo Cruz human subject research board, and all officials responsible provided written informed consent, in accordance with the Declaration of Helsinki of 1975, as revised in 2000 .

\section{Laboratory Methods}

Clinical laboratory analyses were performed in the Clinical Analyses Laboratory of the PHAR-UFBA and the Pathology and Molecular Biology Laboratory of the Centro de Pesquisas Goncalo Moniz da Fundação de Pesquisa Oswaldo Cruz. Biochemical markers analyses were measured in serum by immunochemistry assay (A25 system, BIOSYSTEMS SA, Barcelona, Spain). Serum ferritin was measured by immunoassay using an Access ${ }^{\circ}$ 2 Immunoassay system X2 (Beckman Coulter, Fullerton, CA). C-reactive protein (CRP), alpha 1 -antitripsin (A1AT) and antistreptolysin-O (ASO) were measured by immunochemistry (Immage 800 system, Beckman Coulter, Fullerton, CA). Hematological analyses were carried out using an electronic cell counter, Coulter Count T-890 (Coulter Corporation, FL, USA). The hemoglobin $\mathrm{Hb}$ ) profile and $\mathrm{HbF}$ levels were investigated by high performance liquid chromatography (HPLC/VARIANT I; BIO-RAD, CA, USA).

\section{Definition of Clinical Events}

Clinical data were collected from patient medical records. Demographic data were provided by interviews with patients and parents or guardians. Eligibility criteria included only SCD patients of pediatric age. All patients were in the steady-state of the disease when samples were collected; steady-state was characterized as a period without any acute events and no blood transfusion for 120 days prior to blood sampling. Exclusion criteria included infection or inflammatory episodes and previous blood transfusion (within four months prior to the study). To identify possible associations between HDL-C levels and clinical characteristics in SCD we assessed medical history from patients' records, including prevalence of stroke, number of hospitalizations, painful episodes, VOE, infection, pneumonia, priapism, splenomegaly, splenic sequestration, leg ulcers, cardiac 
abnormalities, respiratory insufficiency and cholelithiasis. Pneumonia was defined as an acute infection of the lung by virus, bacteria or atypical organisms with a clinical outcome that did not meet the criteria for ACS [8].

\section{Statistical analysis}

Baseline characteristics were summarized as means and proportions of selected variables. Distribution of quantitative variables was determined using the KolmogorovSmirnov test. Mean values of quantitative variables between groups were compared using the unpaired t-test for normal data distribution and Mann-Whitney for non-normal data. Bivariate correlation analyses were carried out to determine correlations between pairs of variables using Pearson's and Spearman's rank correlation (R). The nonparametric Kruskal-Wallis test was used to compare means among two or more groups as measured by interval variables. The level of $40 \mathrm{mg} / \mathrm{dl}$ was considered as a reference range and interactions between low HDL-C (less than $40 \mathrm{mg} / \mathrm{dl}$ ) and high HDL-C (at least $40 \mathrm{mg} / \mathrm{dl}$ ) and baseline characteristics were evaluated using independent t-test and MannWhitney tests. The interactions between low HDL-C (less than $40 \mathrm{mg} / \mathrm{dl}$ ) and high HDL-C (at least $40 \mathrm{mg} /$ dl) and specific categorical clinical variables were tested for significance using a $\chi^{2}$ test or Fisher's exact test, taking into account the expected frequency in the cell tables. All tests were considered significant if $p$ values were less than .05. Data analyses were performed using Prism 5.01 (Graphpad Software, San Diego, CA), EPIinfo 6.04 (CDC, Atlanta, Georgia) and STATA SE 10 software (StataCorp, Texas, USA).

\section{Results}

First of all we compared the analyses of markers of intravascular hemolysis, hemolysis and hepatic involvement, leukocyte and platelet counts, renal involvement, lipid metabolism, inflammation and $\mathrm{Hb}$ profile in order to establish how much are the difference between those markers between control and patients groups (Table 1).

\section{HDL-C association with markers of hemolysis, inflammation and vascular dysfunction}

The high-density lipoprotein cholesterol was positively correlated with red blood cells (RBC), Hb, hematocrit and total cholesterol and urea concentrations and negatively correlated with hematimetric indexes of mean cell volume $(\mathrm{MCV})$, mean cell hemoglobin $(\mathrm{MCH})$ and mean cell hemoglobin concentration $(\mathrm{MCHC})$; reticulocytes, hemoglobin $\mathrm{S}(\mathrm{HbS})$, hemolysis and hepatic markers, total leukocytes, monocytes and platelets, alanine aminotransferase (ALT), iron and A1AT. However, it was not correlated with LDL-C. Steady state triglycerides were negatively correlated with $\mathrm{RBC}, \mathrm{Hb}$, hematocrit and HDL-C, and positively correlated with $\mathrm{HbS}$, LDH, AST, total bilirubin, platelet, total protein, total cholesterol, and VLDL-C (Table 2).

We next determined whether the levels of HDL-C in SCD group (HDL-C less than $40 \mathrm{mg} / \mathrm{dl}$ vs. $40 \mathrm{mg} / \mathrm{dl}$ or more) showed difference among the laboratorial markers. In the first group, there were $80 \mathrm{HBSS}$ and $23 H B S C$ patients, and in the second group, there were 23 HBSS and 25 HBSC patients. Sickle cell patients with low HDL-C presented lower RBC counts as well as $\mathrm{Hb}$ and hematocrit concentrations than patients from the group with normal HDL-C levels. The low concentration HDL-C group had higher erythroblast, leukocyte, platelet, neutrophil, monocyte and reticulocyte counts and higher iron, AST, total bilirubin, direct bilirubin, indirect bilirubin, $\mathrm{LDH}$ and $\mathrm{A} 1 \mathrm{AT}$ concentrations. There was no difference in LDL-C concentration between the two HDL-C subgroups, but the VLDL-C and triglycerides concentrations were higher in the low HDL-C group (Table 3).

\section{Association of HDL-C with sickle cell disease clinical history}

We assessed possible associations between HDL-C levels and a series of clinical characteristics in SCD medical history, including prevalence of stroke, number of hospitalizations, painful episodes, VOE, infection, pneumonia, priapism, splenomegaly, splenic sequestration, leg ulcers, cardiac abnormalities, respiratory insufficiency and cholelithiasis. To compare these categorical variables with HDL-C concentration, we divided patients into two groups. The low HDL-C group (less than $40 \mathrm{mg} / \mathrm{dl}$ ) comprised 103 sickle cell disease patients $(80 \mathrm{HBSS}$ and 23 HBSC), with an HDL-C range of $16-39 \mathrm{mg} / \mathrm{dl}$ and mean of $28.95 \mathrm{mg} / \mathrm{dl}$. The high HDL-C group (at least $40 \mathrm{mg} / \mathrm{dl}$ ) comprised $48 \mathrm{SCD}$ patients (23 HBSS and $25 \mathrm{HBSC}$ ), with an HDL-C range of 41-85 $\mathrm{mg} / \mathrm{dl}$ and mean of $51.2 \mathrm{mg} / \mathrm{dl}$.

The prevalence of pneumonia $(\mathrm{OR}=2.42,95 \% \mathrm{CI}$ : 1.06-5.53; $\mathrm{P}=0.033)$ and the prevalence of cardiac abnormalities $(\mathrm{OR}=2.88,95 \% \mathrm{CI}: 1.12-7.59, \mathrm{P}=0.025)$ were significantly different between the HDL-C groups. Forty-one children in the low HDL-C group had cardiac abnormalities typical of hemolytic anemia on auscultation. However, among these, 24 had electrocardiograph arrhythmia, and 3 had tricuspid regurgitant jet velocity of at least $2.6 \mathrm{~m} / \mathrm{sec}$, indicating a possible presence of pulmonary hypertension. These results were obtained from previously performed echocardiograms that were not preformed at the same time of the present study. The low HDL-C concentration group underwent more blood transfusions $(\mathrm{OR}=2.52,95 \% \mathrm{CI}$ : 1.11-5.77, $\mathrm{P}=0.025)$. 
Table 1 Patient and control group characteristics

\begin{tabular}{|c|c|c|c|c|c|}
\hline \multirow[b]{2}{*}{ Characteristics } & \multicolumn{2}{|c|}{ Patients } & \multicolumn{2}{|c|}{ Controls } & \multirow[b]{2}{*}{$p$} \\
\hline & $\mathrm{N}$ & Mean \pm SD & $\mathbf{N}$ & Mean \pm SD & \\
\hline Age (Years) & 152 & $9.2 \pm 4.0$ & 132 & $8.7 \pm 3.2$ & \\
\hline \multicolumn{6}{|l|}{ Gender } \\
\hline Male & 82 & $53.9^{*}$ & 68 & $51.5^{*}$ & \\
\hline Female & 70 & $46.1^{*}$ & 64 & $48.5^{*}$ & \\
\hline \multicolumn{6}{|l|}{ Hemoglobins } \\
\hline AA & - & - & 132 & 100.0 & \\
\hline SS & 103 & 67.8 & - & - & \\
\hline SC & 48 & 31.5 & - & - & \\
\hline SD & 01 & 0.7 & - & - & \\
\hline \multicolumn{6}{|l|}{ Hemoglobin } \\
\hline Fetal (\%) & 142 & $7.51 \pm 6.20$ & 130 & $0.47 \pm 0.46$ & $<0.001$ \\
\hline \multicolumn{6}{|l|}{ Hemolysis } \\
\hline $\mathrm{RBC}\left(\times 10^{6} / \mathrm{cu} \mathrm{mm}\right)$ & 152 & $3.24 \pm 0.97$ & 131 & $4.74 \pm 0.39$ & $<0.001$ \\
\hline Hemoglobin (g/dL) & 152 & $8.93 \pm 2.01$ & 131 & $12.83 \pm 1.03$ & $<0.001$ \\
\hline Hematocrit (\%) & 152 & $27.65 \pm 6.20$ & 131 & $38.47 \pm 2.78$ & $<0.001$ \\
\hline Mean Cell Volume (fL) & 152 & $87.44 \pm 10.85$ & 131 & $81.37 \pm 5.16$ & $<0.001$ \\
\hline Mean Cell Hemoglobin (pg) & 152 & $28.29 \pm 3.73$ & 131 & $27.14 \pm 1.95$ & 0.007 \\
\hline Reticulocyte Count (\%) & 140 & $7.61 \pm 4.88$ & 122 & $0.846 \pm 0.256$ & $<0.001$ \\
\hline \multicolumn{6}{|l|}{ Leukocytes } \\
\hline Leukocyte Count $\left(\times 10^{9} / \mathrm{L}\right)$ & 152 & $13.1 \pm 5.8$ & 131 & $7.0 \pm 2.2$ & $<0.001$ \\
\hline Neutrophil Count $\left(\times 10^{9} / \mathrm{L}\right)$ & 152 & $6161.72 \pm 3779.49$ & 131 & $3240.32 \pm 1686.15$ & $<0.001$ \\
\hline Monocyte Count $\left(\times 10^{9} / \mathrm{L}\right)$ & 152 & $817.15 \pm 481.83$ & 131 & $488.67 \pm 204.90$ & $<0.001$ \\
\hline \multicolumn{6}{|l|}{ Platelets } \\
\hline Platelet Count $\left(\times 10^{9} / \mathrm{L}\right)$ & 152 & $403.93 \pm 158.66$ & 131 & $308.21 \pm 67.35$ & $<0.001$ \\
\hline \multicolumn{6}{|l|}{ Lipid metabolism } \\
\hline Total Cholesterol (mg/dL) & 151 & $121.12 \pm 26.16$ & 124 & $164.08 \pm 34.55$ & $<0.001$ \\
\hline HDL Cholesterol (mg/dL) & 151 & $35.65 \pm 12.34$ & 123 & $48.90 \pm 13.67$ & $<0.001$ \\
\hline LDL Cholesterol (mg/dL) & 151 & $64.95 \pm 22.19$ & 123 & $97.41 \pm 33.54$ & $<0.001$ \\
\hline VLDL Cholesterol (mg/dL) & 151 & $20.44 \pm 9.38$ & 123 & $17.75 \pm 10.37$ & $<0.001$ \\
\hline Triglycerides (mg/dL) & 150 & $102.07 \pm 46.86$ & 123 & $88.31 \pm 51.73$ & 0.002 \\
\hline \multicolumn{6}{|l|}{ Hemolysis plus Hepatic } \\
\hline Aspartate aminotransferase (U/L) & 152 & $48.05 \pm 24.92$ & 122 & $30.28 \pm 11.13$ & $<0.001$ \\
\hline Total bilirubin (mg/dL) & 151 & $2.73 \pm 1.76$ & 118 & $0.49 \pm 0.21$ & $<0.001$ \\
\hline Direct bilirubin (mg/dL) & 151 & $0.66 \pm 0.46$ & 118 & $0.250 \pm 0.082$ & $<0.001$ \\
\hline Indirect bilirubin (mg/dL) & 151 & $2.08 \pm 1.59$ & 118 & $0.244 \pm 0.182$ & $<0.001$ \\
\hline Iron serum (mcg/dL) & 126 & $123.40 \pm 119.94$ & 119 & $71.14 \pm 40.31$ & $<0.001$ \\
\hline Lactate dehydrogenase(U/L) & 151 & $858.22 \pm 503.81$ & 119 & $406.27 \pm 132.03$ & $<0.001$ \\
\hline \multicolumn{6}{|l|}{ Hepatic } \\
\hline Alanine aminotransferase $(\mathrm{U} / \mathrm{L})$ & 152 & $28.25 \pm 21.34$ & 121 & $17.36 \pm 7.10$ & $<0.001$ \\
\hline Total protein (g/dL) & 151 & $7.33 \pm 0.848$ & 119 & $7.31 \pm 0.62$ & 0.695 \\
\hline Albumin (g/dL) & 151 & $4.07 \pm 0.675$ & 119 & $4.24 \pm 0.49$ & 0.249 \\
\hline Globulin (g/dL) & 151 & $3.26 \pm 0.781$ & 119 & $3.06 \pm 0.63$ & 0.109 \\
\hline Albumin/Globulin ratio & 151 & $1.35 \pm .54$ & 112 & $1.44 \pm .42$ & 0.289 \\
\hline \multicolumn{6}{|l|}{ Renal } \\
\hline Urea nitrogen (mg/dL) & 150 & $17.73 \pm 6.41$ & 120 & $21.65 \pm 5.92$ & $<0.001$ \\
\hline Creatinine $(\mathrm{mg} / \mathrm{dL})$ & 151 & $0.51 \pm 0.50$ & 120 & $0.523 \pm 0.185$ & 0.708 \\
\hline \multicolumn{6}{|l|}{ Inflammation } \\
\hline C-reactive protein (mg/L) & 148 & $7.08 \pm 11.97$ & 102 & $2.01 \pm 2.29$ & $<0.001$ \\
\hline Alpha 1-antitrypsin (mg/dL) & 151 & $152.50 \pm 46.18$ & 129 & $137.48 \pm 43.36$ & 0.013 \\
\hline Ferritin (ng/mL) & 152 & $313.32 \pm 361.44$ & 117 & $37.29 \pm 28.28$ & $<0.001$ \\
\hline Antistreptolysin-O(UI/mL) & 148 & $192.70 \pm 285.42$ & 101 & $132.75 \pm 131.19$ & 0.181 \\
\hline
\end{tabular}

* percentage Mann-Whitney test 
Table 2 Laboratory value associations with HDL-C and Triglycerides in sickle cell disease

\begin{tabular}{|c|c|c|c|c|}
\hline & \multicolumn{2}{|c|}{$\begin{array}{l}\text { HDL Cholesterol } \\
\text { (mg/dL) }\end{array}$} & \multicolumn{2}{|c|}{$\begin{array}{c}\text { Triglycerides } \\
\text { (mg/dL) }\end{array}$} \\
\hline & $r$ & $p$ & $r$ & $p$ \\
\hline \multicolumn{5}{|l|}{ Hemoglobin } \\
\hline S hemoglobin (\%) & -0.311 & 0.002 & 0.286 & 0.005 \\
\hline Fetal hemoglobin (\%) & -0.048 & 0.644 & -0.685 & 0.685 \\
\hline \multicolumn{5}{|l|}{ Hemolysis } \\
\hline $\mathrm{RBC}\left(\times 10^{6} / \mathrm{cu} \mathrm{mm}\right)$ & 0.328 & $<0.001$ & -0.190 & 0.019 \\
\hline Hemoglobin (g/dL) & 0.292 & $<0.001$ & -0.202 & 0.013 \\
\hline Hematocrit (\%) & 0.309 & $<0.001$ & -0.189 & 0.020 \\
\hline Mean Cell Volume (fL) & $-0.273 \mathbf{4}$ & 0.006 & 0.126 & 0.125 \\
\hline Mean Cell Hemoglobin (pg) & -0.284 & 0.002 & 0.111 & 0.175 \\
\hline Reticulocyte Count (\%) & -0.170 & 0.046 & 0.082 & 0.339 \\
\hline \multicolumn{5}{|l|}{ Leukocyte } \\
\hline Leukocyte Count $\left(\times 10^{9} / \mathrm{L}\right)$ & -0.198 & 0.015 & 0.081 & 0.325 \\
\hline Neutrophil Count $\left(\times 10^{9} / \mathrm{L}\right)$ & $0.017 \notin$ & 0.838 & -0.154 & 0.061 \\
\hline Monocyte Count $\left(\times 10^{9} / \mathrm{L}\right)$ & -0.234 & 0.004 & 0.139 & 0.089 \\
\hline \multicolumn{5}{|l|}{ Platelets } \\
\hline Platelet Count $\left(\times 10^{9} / \mathrm{L}\right)$ & -0.228 & 0.005 & 0.233 & 0.004 \\
\hline \multicolumn{5}{|l|}{ Hemolysis plus Hepatic } \\
\hline Aspartate aminotransferase (U/L) & -0.235 & 0.004 & 0.207 & 0.011 \\
\hline Total bilirubin (mg/dL) & -0.298 & $<0.001$ & 0.165 & 0.044 \\
\hline Direct bilirubin (mg/dL) & -0.471 & $<0.001$ & 0.035 & 0.669 \\
\hline Indirect bilirubin (mg/dL) & -0.287 & $<0.001$ & 0.140 & 0.088 \\
\hline Iron Serum (mcg/dL) & -0.186 & 0.038 & 0.159 & 0.076 \\
\hline Lactate dehydrogenase (U/L) & -0.375 & $<0.001$ & 0.167 & 0.041 \\
\hline \multicolumn{5}{|l|}{ Hepatic } \\
\hline Alanine aminotransferase $(\mathrm{U} / \mathrm{L})$ & -0.172 & 0.035 & 0.075 & 0.364 \\
\hline Total protein (g/dL) & $-0.021+$ & 0.793 & 0.274 & 0.001 \\
\hline Albumin (g/dL) & 0.102 & 0.213 & 0.142 & 0.083 \\
\hline Globulin (g/dL) & $-0.124 \uparrow$ & 0.129 & 0.133 & 0.104 \\
\hline Albumin/Globulin ratio & 0.033 & 0.689 & -0.033 & 0.684 \\
\hline \multicolumn{5}{|l|}{ Renal } \\
\hline Urea nitrogen (mg/dL) & 0.178 & 0.030 & 0.020 & 0.806 \\
\hline Creatinine (mg/dL) & 0.118 & 0.152 & 0.105 & 0.201 \\
\hline \multicolumn{5}{|l|}{ Lipid metabolism } \\
\hline Total Cholesterol (mg/dL) & 0.299 & $<0.001$ & 0.268 & 0.001 \\
\hline HDL Cholesterol (mg/dL) & - & - - & -0.228 & 0.005 \\
\hline LDL Cholesterol (mg/dL) & -0.083 & 0.312 & 0.068 & 0.409 \\
\hline VLDL Cholesterol (mg/dL) & -0.242 & 0.003 & 0.998 & $<0.001$ \\
\hline Triglycerides (mg/dL) & -0.228 & 0.005 & - & - \\
\hline \multicolumn{5}{|l|}{ Inflammation } \\
\hline C-reactive protein $(\mathrm{mg} / \mathrm{L})$ & 0.048 & 0.563 & -0.031 & 0.714 \\
\hline Alpha 1 antitrypsin (mg/dL) & -0.327 & $<0.001$ & -0.074 & 0.378 \\
\hline Ferritin $(\mathrm{ng} / \mathrm{mL})$ & -0.032 & 0.699 & 0.102 & 0.220 \\
\hline Antistreptolysin $\mathrm{O}(\mathrm{UI} / \mathrm{mL})$ & -0.079 & 0.339 & 0.157 & 0.058 \\
\hline
\end{tabular}

Spearman or Pearson correlation coefficients $(r)$ and $p$ values $(p) \& r=$ Pearson correlation coefficient
High serum levels of LDL-C, VLDL-C and total cholesterol lipoproteins, TRIG, ferritin and A1AT but not HDL-C were associated with the occurrence of cholelithiasis (Figure 1).

\section{Discussion}

The present study analyzed levels of HDL-C in steadystate children with SCD. Children with SCD, even in steady-state, have differences in several biomarkers as compared to healthy age-matched children [13]. Those differences are related to numerous mechanisms associated with infection, inflammation and VOE in the disease $[1,2]$. Several biomarkers associated with hemolysis, inflammation, renal metabolism, hepatic metabolism, and lipid metabolism in children with SCD and healthy subjects were studied, and the findings of normal concentrations of protein and globulin as well as the albu$\mathrm{min} /$ globulin ratio among the SCD patients suggest an absence of early severe liver cell damage in the studied group [13]. Normal levels of creatinine in the patient group confirm previous observations that an increased rate of creatinine secretion by dysfunctional renal tubules may lead to a falsely normal plasma creatinine and creatinine clearance. A more accurate evaluation of different aspects of SCD nephropathy, emphasizing proteinuria and hyperfiltration, needs to be developed in children in order to detect early renal alteration [14-16].

Hypocholesterolemia has been described in SCD patients with significantly decreased LDL-C and HDL-C [17-22] and has been also described for our group as a potential biomarker for SCD clinical severity [23]. A negative association was found for HDL-C and VLDL-C, which was directly associated with triglycerides. Triglyceride-rich VLDL-C particles availability may play an important role in lipid oxidization in SCD patients. It has been suggested that VLDL-C is an important factor for atherosclerosis development. VLDL-C particles assemble by a complex process that includes an apolipoprotein $\mathrm{B}$ (apoB)-containing VLDL precursor and a VLDL-sized lipid droplet lacking apoB. Both particles fuse to produce a mature VLDL particle [24]. The increase of triglycerides probably contributes to an increase in the hepatic production of VLDL-C, increasing the number of receptors for LDL-C that is extensively metabolized, decreasing its serum levels. However, the role of cholesterol and triglycerides and the regulation of assembly and production of VLDL-C are poorly understood.

A negative association was observed between $\mathrm{LDH}$ and HDL-C, showing that HDL-C, as measured by its 
Table 3 Laboratory values for sickle cell disease patients with different steady-state levels of HDL-C

\begin{tabular}{|c|c|c|c|c|c|}
\hline & \multicolumn{2}{|c|}{${ }^{* *} \mathrm{HDL}$ less than $40 \mathrm{mg} / \mathrm{dL}$} & \multicolumn{2}{|c|}{${ }^{* * *} \mathrm{HDL}$ at least $40 \mathrm{mg} / \mathrm{dL}$} & \multirow[b]{2}{*}{$* p$} \\
\hline & $\mathbf{N}$ & Mean \pm SD & $\mathrm{N}$ & Mean \pm SD & \\
\hline \multicolumn{6}{|l|}{ Hemolysis } \\
\hline $\mathrm{RBC}\left(\times 10^{6} / \mathrm{cu} \mathrm{mm} \mathrm{L}\right)$ & 103 & $3.01 \pm 0.85$ & 48 & $3.75 \pm 1.0$ & $<0.001$ \\
\hline Hemoglobin (g/dL) & 103 & $8.57 \pm 2.02$ & 48 & $9.76 \pm 1.75$ & 0.001 \\
\hline Hematocrit (\%) & 103 & $26.42 \pm 6.17$ & 48 & $30.43 \pm 5.37$ & $<0.001$ \\
\hline Mean Cell Volume (fL) & 103 & $89.17 \pm 10.34$ & 48 & $83.52 \pm 11.01$ & 0.003 \\
\hline Mean Cell Hemoglobin (pg) & 103 & $28.94 \pm 3.52$ & 48 & $26.82 \pm 3.79$ & 0.001 \\
\hline Mean Cell Hemoglobin Concentration (\%) & 103 & $32.44 \pm 0.96$ & 48 & $32.07 \pm 0.86$ & 0.025 \\
\hline Erythroblast (\%) & 103 & $1.90 \pm 2.31$ & 48 & $1.02 \pm 2.48$ & 0.034 \\
\hline Reticulocyte count (\%) & 97 & $8.34 \pm 4.55$ & 42 & $5.90 \pm 5.25$ & 0.006 \\
\hline \multicolumn{6}{|l|}{ Hemoglobins } \\
\hline S hemoglobin (\%) & 97 & $79.22 \pm 16.16$ & 44 & $60.75 \pm 18.22$ & $<0.001$ \\
\hline Fetal hemoglobin (\%) & 97 & $7.55 \pm 5.99$ & 44 & $7.41 \pm 6.78$ & 0.899 \\
\hline \multicolumn{6}{|l|}{ Leukocyte } \\
\hline Leukocyte Count $\left(\times 10^{9} / \mathrm{L}\right)$ & 103 & $14105.83 \pm 6085.37$ & 48 & $10868.75 \pm 4416.13$ & 0.001 \\
\hline Neutrophil Count $\left(\times 10^{9} / \mathrm{L}\right)$ & 103 & $6723.36 \pm 4167.75$ & 48 & $4971.83 \pm 2459.10$ & 0.002 \\
\hline Monocyte Count $\left(\times 10^{9} / \mathrm{L}\right)$ & 103 & $910.35 \pm 499.84$ & 48 & $604.69 \pm 361.83$ & 0.004 \\
\hline \multicolumn{6}{|l|}{ Platelets } \\
\hline Platelet Count $\left(\times 10^{9} / \mathrm{L}\right)$ & 103 & $424.90 \pm 160.26$ & 48 & $357.69 \pm 148.02$ & 0.015 \\
\hline \multicolumn{6}{|l|}{ Hemolysis plus Hepatic } \\
\hline Aspartate aminotransferase (U/L) & 103 & $51.45 \pm 26.29$ & 48 & $40.65 \pm 20.32$ & 0.013 \\
\hline Total bilirubin (mg/dL) & 103 & $3.13 \pm 1.82$ & 48 & $1.88 \pm 1.25$ & $<0.001$ \\
\hline Direct bilirubin (mg/dL) & 103 & $0.79 \pm 0.47$ & 48 & $0.38 \pm 0.25$ & $<0.001$ \\
\hline Indirect bilirubin (mg/dL) & 103 & $2.34 \pm 1.68$ & 48 & $1.50 \pm 1.20$ & 0.002 \\
\hline Iron serum (mcg/dL) & 95 & $136.65 \pm 133.77$ & 31 & $82.77 \pm 40.17$ & 0.029 \\
\hline Lactate dehydrogenase $(\mathrm{U} / \mathrm{L})$ & 103 & $977.19 \pm 524.50$ & 48 & $602.92 \pm 339.59$ & $<0.001$ \\
\hline \multicolumn{6}{|l|}{ Lipid metabolism } \\
\hline Total Cholesterol (mg/dL) & 103 & $116.49 \pm 25.17$ & 48 & $131.06 \pm 25.73$ & 0.001 \\
\hline LDL Cholesterol (mg/dL) & 103 & $65.78 \pm 21.47$ & 48 & $63.19 \pm 23.81$ & 0.506 \\
\hline VLDL Cholesterol (mg/dL) & 103 & $21.62 \pm 10.31$ & 48 & $17.90 \pm 6.38$ & 0.023 \\
\hline Triglycerides (mg/dL) & 102 & $107.74 \pm 51.64$ & 48 & $90.02 \pm 31.86$ & 0.030 \\
\hline \multicolumn{6}{|l|}{ Hepatic } \\
\hline Alanine aminotransferase $(\mathrm{U} / \mathrm{L})$ & 103 & $29.90 \pm 22.10$ & 48 & $24.58 \pm 19.56$ & 0.156 \\
\hline Total protein $(\mathrm{g} / \mathrm{dL})$ & 103 & $7.40 \pm .89$ & 48 & $7.46 \pm .82$ & 0.684 \\
\hline Albumin (g/dL) & 103 & $4.01 \pm .75$ & 48 & $4.23 \pm .59$ & 0.054 \\
\hline Globulin (g/dL) & 103 & $3.36 \pm .85$ & 48 & $3.23 \pm .72$ & 0.333 \\
\hline Albumin/Globulin ratio & 103 & $1.34 \pm .55$ & 48 & $1.38 \pm .53$ & 0.709 \\
\hline \multicolumn{6}{|l|}{ Renal } \\
\hline Urea nitrogen (mg/dL) & 102 & $17.25 \pm 6.70$ & 47 & $18.77 \pm 5.73$ & 0.181 \\
\hline Creatinine (mg/dL) & 103 & $0.49 \pm 0.50$ & 47 & $0.57 \pm 0.50$ & 0.315 \\
\hline \multicolumn{6}{|l|}{ Inflammation } \\
\hline C-reactive protein (mg/L) & 101 & $7.79 \pm 14.02$ & 46 & $5.39 \pm 5.18$ & 0.133 \\
\hline Alpha 1 antitrypsin (mg/dL) & 102 & $163.13 \pm 44.06$ & 48 & $128.92 \pm 42.13$ & $<0.001$ \\
\hline Ferritin (ng/mL) & 103 & $300.76 \pm 399.59$ & 46 & $323.46 \pm 348.87$ & 0.740 \\
\hline Antistreptolysin $\mathrm{O}(\mathrm{UI} / \mathrm{mL})$ & 101 & $198.62 \pm 288.98$ & 46 & $183 \pm 282.56$ & 0.759 \\
\hline
\end{tabular}

* unpaired t-test ** 80 HBSS and 23 HBSC ***23 HBSS and 25 HBSC 


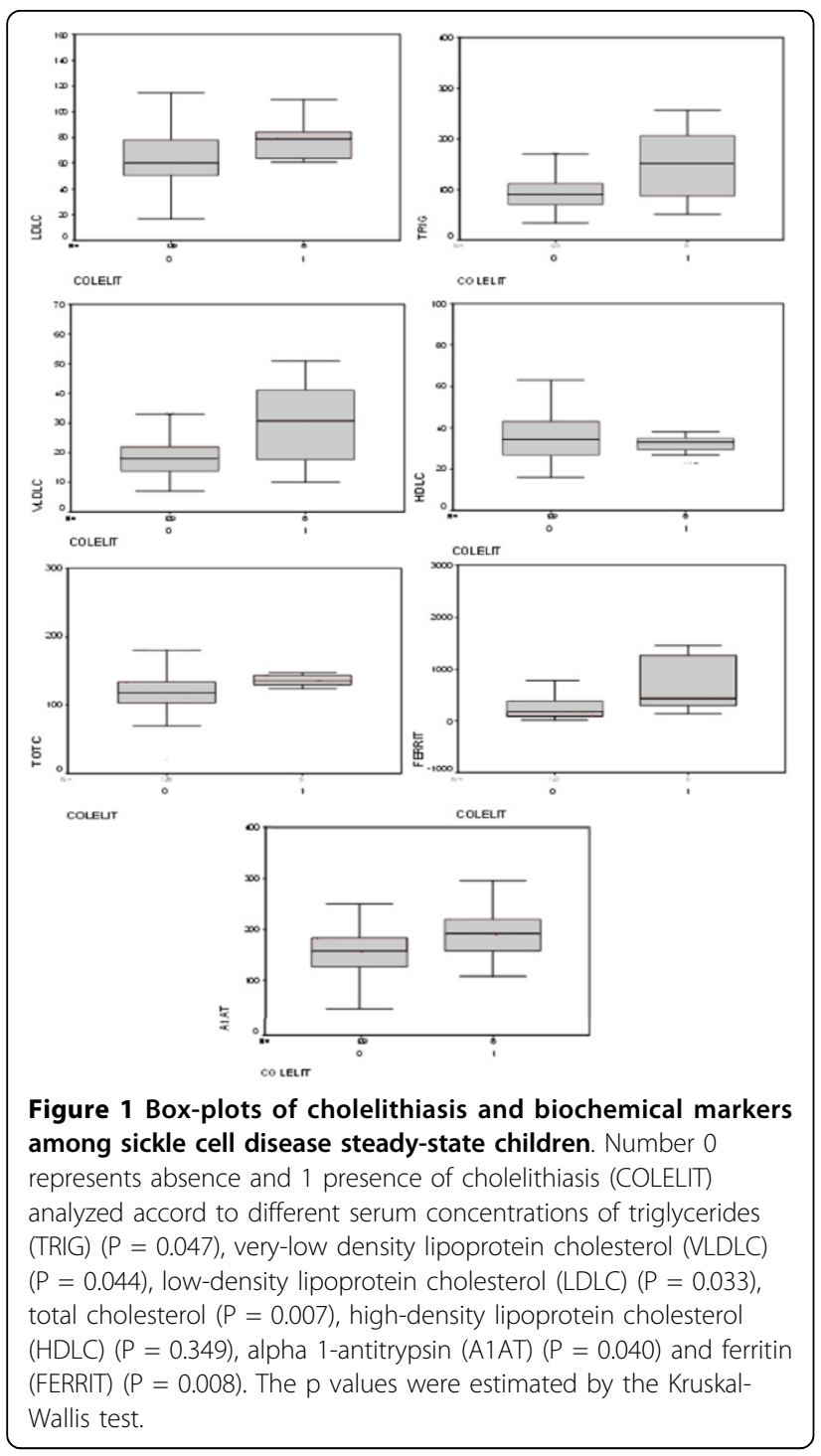

concentration, may function as a prognostic marker of intravascular hemolysis and endothelial dysfunction given its anti-inflammatory, anti-oxidative, anti-aggregation, anti-coagulant and pro-fibrinolytic activities $[25,26]$.

Sickle cell disease patients with higher HDL-C levels presented a low risk of hemolysis and endothelial dysfunction, including lower reticulocyte and erythroblast counts as well as a lower $\mathrm{HbS}$ concentration and it may be related to the high consumption of cholesterol due to acceleration of blood marrow cell production during hemolysis crisis. Sickle cell disease patients with higher HDL-C levels had lower leukocyte, monocyte and platelet counts as well as a lower concentration of hepatic and hemolytic markers and significantly lower VLDL-C, triglycerides and A1AT concentrations; this may reflect the action of the anti-inflammatory and anti-oxidative properties of this biomarker $[25,26]$. The high-density lipoprotein cholesterol removes excess cholesterol from peripheral tissues and transports it to the liver for excretion via bile by reverse cholesterol transport. The highdensity lipoprotein is made up of several particles with different composition and function [24,27,28].

Further confirmation of these associations came from comparing HDL-C concentrations and patients' clinical records, which revealed a higher occurrence of pneumonia and cardiac abnormalities among those with lower HDL-C levels. The results related to pneumonia risk can be explained by the production of auto-antibodies specific to oxidized phospholipids; these autoantibodies have been shown to inhibit macrophage uptake of oxidized LDL and to provide protection against virulent pneumococcal infection [29]. Low levels of HDL-C are an important cardiovascular risk factor, and HDL-C and apoA-I have been shown to decrease lesions and improve vascular reactivity in animal models of atherosclerosis and in humans; these changes may be due to the reduction of oxidized lipids and the enhancement of reverse cholesterol transport [30]. The presence of pulmonary hypertension was shown to be associated with several laboratory test alterations [31]. Recent study has also demonstrated the important role of the apolipoprotein pathway and its association with endothelial dysfunction in SCD patients with pulmonary hypertension [31].

Patients with lower HDL-C levels were also likely to have had more blood transfusions; this can be linked with a more severe clinical course of disease, once that it is a therapeutic strategy used to prevent several clinical symptoms, such as stroke [1].

It is well known that gallstones in patients with hemolytic anemia are said to be calcium bilirubinate stones. In view our results of correlation of cholesterol and triglycerides with hemolysis, we propose that the stones in SCD patients could be related directly to hemolysis and bilirubin generation, and indirectly to cholesterol and lipids and it could be a novel observation and needs to be confirmed by further studies. The association of acute-phase proteins and cholelithiasis may be explained by the response to stress due to traumatic injury or infection-related mechanisms including hypermetabolism and protein catabolism associated with a cytokinedriven inflammatory response.

\section{Conclusions}

In conclusion, we hypothesize that some SCD patients can have a specific dyslipidemic subphenotype characterized by low HDL-C with hypertriglyceridemia and high VLDL-C in association with other biomarkers, including those related to inflammation. This represents an important step toward a more reliable clinical 
prognosis. Additional studies are warranted to test this hypothesis and the probably mechanisms involved in this complex network of markers and their role in SCD pathogenesis.

\begin{abstract}
Abbreviations
SCD: Sickle cell disease; VOE: vaso-occlusive pain episodes; HDL-C: highdensity lipoprotein cholesterol; LDL-C: low-density lipoprotein cholesterol; VLDL-C: very low-density lipoprotein cholesterol; LDH: lactate dehydrogenase; CRP: C-reactive protein; A1AT: alpha 1-antitripsin; ASO: antistreptolysin-O; HB: hemoglobin; HBF: hemoglobin fetal; HPLC: high performance liquid chromatography; RBC: red blood cells; MCV: mean cell volume; MCH: mean cell hemoglobin; MCHC: mean cell hemoglobin concentration; HBS: hemoglobin S; COLELIT: cholelithiasis.
\end{abstract}

\section{Acknowledgements}

The authors would like to thank the subjects from HEMOBA and PHAR-UFBA who participated in the study. They are also grateful for the expertise provided by the staff of FIOCRUZ, HEMOBA and PHAR-UFBA. This work was supported by grants from the Brazilian National Council of Research (CNPq) (3065427/2007-5 and 484457/2007-1) (M.S.G.); the Foundation of Research and Extension of Bahia (FAPESB) (1431040053063 and 9073/2007) (M.S.G.); and MCD/CNPq/MS-SCTIE-DECIT (409800/2006-6), (M.S.G.). The sponsors of this study are public or nonprofit organizations that support science in general. They had no role in gathering analyzing, or interpreting the data.

\section{Author details}

'Laboratório de Patologia e Biologia Molecular, Centro de Pesquisa Gonçalo Moniz, Fundação de Pesquisa Oswaldo Cruz (FIOCRUZ), Salvador, Bahia, Brasil. ${ }^{2}$ Departamento de Análises Clínicas e Toxicológicas, Faculdade de Farmácia, Universidade Federal da Bahia, Salvador, Bahia, Brasil. ${ }^{3}$ Fundação de Hematologia e Hemoterapia do Estado da Bahia (HEMOBA), Salvador, Bahia, Brasil. ${ }^{4}$ Hospital Pediátrico Professor Hosannah de Oliveira, Universidade Federal da Bahia, Salvador, Bahia, Brasil.

\section{Authors' contributions}

MOS performed experiments and analyzed the results; $L R, M B C, J M, R D C$, and AMA performed experiments; IML, VMLN and LR performed clinical evaluation of patients; MGR analyzed the results; and MSG was the principal investigator and takes primary responsibility for the paper, designed the research, analyzed the results and wrote the paper. All authors read and approved the final manuscript.

\section{Competing interests}

The authors declare that they have no competing interests.

Received: 12 July 2010 Accepted: 27 August 2010

Published: 27 August 2010

\section{References}

1. Ohene-Frempong K, Steinberg MH: Clinical Aspects of sickle cell anemia in adults and children. In Disorders of Hemoglobin: Genetics, In Patophysiology, and Clinical Management. Edited by: Steinberg MH, Forget BG, Higgs DR, Nagel RL. New York: Cambridge University Press; 2001:611-70.

2. Stuart MJ, Nagel RL: Sickle cell disease. Lancet 2004, 364:1343-60.

3. Nagel RL, Platt OS: General pathophysiology of sickle cell anemia. In Disorders of Hemoglobin: Genetics, In Pathophysiology, and Clinical Management. Edited by: Steinberg MH, Forget BG, Higgs DR, Nagel RL. New York: Cambridge University Press; 2001:494-526.

4. Sebastiani P, Nolan VG, Baldwin $C T$, Abad-Grau MM, Wang L, Adewoye AH, McMahon LC, Farrer LA, Taylor JG, Kato GJ, Gladwin MT, Steinberg MH: A network model to predict the risk of death in sickle cell disease. Blood 2007, 110:2727-2763.

5. Okpala I: The intriguing contribution of white blood cells to sickle cell disease- a red cell disorder. Blood Reviews 2004, 18:65-73.

6. Telen MJ: Role of adhesion molecules and vascular endothelium in the pathogenesis of sickle cell disease. Hematology Am Soc of Hematol Educ Program 2007, 2007:84-90.
7. Villagra J, Shiva S, Hunter LA, Machado RF, Gladwin MT, Kato GJ: Platelet activation in patients with sickle cell disease, hemolysis-associated pulmonary hypertension, and nitric oxide scavenging by cell-free hemoglobin. Blood 2007, 110:2166-2172.

8. Johnson C, Telen MJ: Adhesion molecule and hydroxyurea in the pathophysiology of sickle cell disease. Hematologica 2008, 93:481-486.

9. Gladwin MT, Crawford JH, Patel RP: Serial review: Biomedial implications for hemoglobin interactions with nitric oxide. Free Radical Biology \& Medicine 2003, 36:707-717.

10. Kato GJ, McGowan V, Machado RF, Little JA, Taylor J, Morris CR, Nichols JS, Wang X, Poljakovic M, Morris SM Jr, Gladwin MT: Lactate dehydrogenase as a biomarker of hemolysis-associated nitric oxide resistance, priapism, leg ulceration, pulmonary hypertension, and death in patients with sickle cell disease. Blood 2006, 107:2279-2285.

11. O'Driscoll S, Height SE, Dick MC, Rees DC: Serum lactate dehydrogenase activity as a biomarker in children with sickle cell disease. $\mathrm{Br} J$ Haematol 2007, 140:206-209.

12. Azevêdo ES: Subgroup studies of black admixture within a mixed population of Bahia, Brazil. Ann Hum Genet 1980, 44:55-60.

13. Isichei UP: Liver function and the diagnosis significance of biochemical changes in the blood of African children with sickle cell disease. J Clin Pathol 1980, 33:626-630.

14. Skalar AH, Campbell H, Caruana RJ, Lightfoot BO, Gaier JG, Milner P: A population study of renal function in sickle cell anemia. Int J Artif Organs 1990, 13:231-236.

15. Skalar AH, Perez JC, Harp RJ, Caruana LRJ: Acute renal failure in sickle cell anemia. Int J Artif Organs 1990, 13:347-351.

16. Marouf R, Mojiminiyi O, Abdella N, Kortom M, Al Wazzan H: Comparison of renal function markers in Kuwaiti patients with sickle cell disease. J Clin Pathol 2006, 59:345-351.

17. Sasaki J, Waterman MR, Buchanan GR, Cottam GL: Plasma and erythrocyte lipids in sickle cell anaemia. Clin Lab Haematol 1983, 5:35-44.

18. VanderJagt DJ, Huang YS, Chuang LT: Phase angle and $n-3$ polyunsaturated fatty acids in sickle cell disease. Arch Dis Child 2002, 87:252-254.

19. VanderJagt DJ, Shores J, Okorodudu A, Okolo SN, Glew RH: Hypocholesterolemia in Nigerian children with sickle cell disease. J Trop Pediatr 2002, 48:156-161.

20. Shores J, Peter son J, Vanderjagt D, Gless RH: Reduced cholesterol levels in African-American adults with sickle cell disease. J Natl Med Assoc 2003, 95:813-817.

21. Djoumessi S, Zekeng L, Lando G, Zeukeng D: Serum lipids and atherogenic risk in sickle- cell trait carriers. Ann Biol Clin 1994, 52:663-665.

22. Zorca S, Freeman L, Hildesheim M, Allen D, Remaley AT, Taylor JG, Kato GJ: Lipid levels in sickle-cell disease associated with haemolytic severity, vascular dysfunction and pulmonary hypertension. Br J Haematol 2010, 149:436-45.

23. Magda Oliveira Seixas, Larissa Rocha, Mauricio Carvalho, Joelma Menezes, Isa Lyra, Valma Nascimento, Ricardo Couto, Ajax Atta, Mitermayer Galvão Reis, Marilda Souza Goncalves: Lipoprotein Cholesterol and Triglycerides in Children with steady-state sickle cell anemia [Abstract]. Blood 2009, 114:s1547.

24. Prinsen BHCMT, Romiju JA, Bisschop PH: Endogenous cholesterol synthesis is associated with VLDL-2 apo-B100 production in healthy humans. $J$ Lipid Res 2003, 44:1341-1348.

25. Nofer JR, Kehrel B, Fobker M, Levkau B, Assmann G, von Eckardstein A: HDL arteriosclerosis: beyond reverse cholesterol transport. Atherosclerosis 2002, 161:1-16.

26. Fredenrich A, Bayer P: Reverse cholesterol transport, high density lipoproteins and HDL cholesterol: recent data. Diabetes Metab 2003, 29:201-205.

27. Miyazaki O, Fukamavhi I, Mori A: Formation of pre $\beta 1-H D L$ during lipolysis of triglyceride-rich lipoprotein. Biochem Biophys Res Commun 2009, 379:55-59.

28. Pearson T, Wattis JA, O'Malley B: Mathematical modeling of competitive LDL/VLDL binding and update by hepatocytes. J Math Biol 2009, 58:845-880.

29. Navab M, Berliner JA, Subbanagounder G, Hama S, Lusis AJ, Castellani LW, Reddy S, Shih D, Shi W, Watson AD, Van Lenten BJ, Vora D, Fogelman AM: $\mathrm{HDL}$ and inflammatory response induced by LDL-derived oxidized phospholipids. Arterioscler Thromb Vasc Biol 2001, 21:481-488. 
30. Navab M, Ananthramaiah GM, Reddy ST: The oxidation hypothesis hypothesis of atherogenesis: the role of oxidized phospholipids and HDL. J Lipid Res 2004, 45:9932-1007.

31. Minniti CP, Sable C, Campbell A, Rana S, Ensing G, Dham N, Onyekwere O, Nouraie M, Kato GJ, Gladwin MT, Castro OL, Gordeuk VR: Elevated tricuspid regurgitant jet velocity in children and adolescents with sickle cell disease: association with hemolysis and hemoglobin oxygen desaturation. Haematologica 2009, 94:340-347.

doi:10.1186/1476-511X-9-91

Cite this article as: Seixas et al:: Levels of high-density lipoprotein cholesterol (HDL-C) among children with steady-state sickle cell disease. Lipids in Health and Disease 2010 9:91.

Submit your next manuscript to BioMed Central and take full advantage of:

- Convenient online submission

- Thorough peer review

- No space constraints or color figure charges

- Immediate publication on acceptance

- Inclusion in PubMed, CAS, Scopus and Google Scholar

- Research which is freely available for redistribution

Submit your manuscript at www.biomedcentral.com/submit 